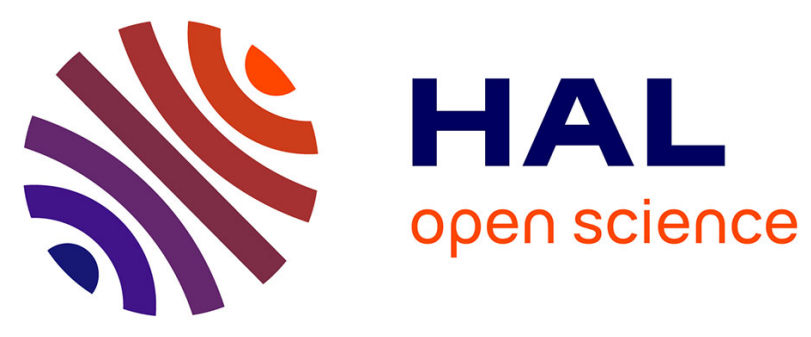

\title{
First experimental demonstration of real-time orchestration in a Multi-head metro network
}

Lida Sadeghioon, Paulette Gavignet, Ahmed Triki, Jean-Luc Barbey, Esther

Le Rouzic, Laurent Bramerie, Vincent Alaiwan, Eric Borgne, Christophe

Betoule, Bernard Arzur, et al.

\section{To cite this version:}

Lida Sadeghioon, Paulette Gavignet, Ahmed Triki, Jean-Luc Barbey, Esther Le Rouzic, et al.. First experimental demonstration of real-time orchestration in a Multi-head metro network. 16th International Conference on Transparent Optical Networks (ICTON 2014), Jul 2014, Graz, Austria. pp.1 4, 10.1109/ICTON.2014.6876667 . hal-01084900

\section{HAL Id: hal-01084900 https://hal.science/hal-01084900}

Submitted on 20 Nov 2014

HAL is a multi-disciplinary open access archive for the deposit and dissemination of scientific research documents, whether they are published or not. The documents may come from teaching and research institutions in France or abroad, or from public or private research centers.
L'archive ouverte pluridisciplinaire HAL, est destinée au dépôt et à la diffusion de documents scientifiques de niveau recherche, publiés ou non, émanant des établissements d'enseignement et de recherche français ou étrangers, des laboratoires publics ou privés. 


\title{
First Experimental Demonstration of Real-Time Orchestration in a Multi-Head Metro Network
}

\author{
Lida Sadeghioon, Paulette Gavignet, Ahmed Triki, Jean-Luc Barbey, Esther Le Rouzic, \\ Laurent Bramerie*, Vincent Alaiwan*, Eric Borgne*, Christophe Betoule, Bernard Arzur, \\ Arnaud Carer* \\ Orange Labs, 22307 Lannion, France \\ * University Rennes I, Lannion, France \\ e-mail:Lida.Mollazadehsadeghioon@Orange.com
}

\begin{abstract}
We present for the first time the experimental demonstration of a Real-Time control-plane on the Multi-hEad sub-wavElength swiTching (MEET), Metro architecture. The key control assets are calculated and provided to the edge nodes in a form of grant files. These grant files eliminate the contention possibility at source nodes and destinations, thus they offer a lossless passive optical grooming and multiplexing/demultiplexing at the intermediate nodes. The experimental results validate the control plane structure designed based on a deterministic operating system well scalable for a regional metro network.
\end{abstract}

Keywords: Time-domain Wavelength Interleaved Networking, Burst switching, Centralised control plane, Metropolitan Area Network,

\section{INTRODUCTION}

The advances in optical transmission technology mobilize the surge of channel bit-rate everywhere within the telecommunication networks. However there still remains the challenge of filling these growing size telecommunication pipes with multiple granular sizes of traffic, since the studies conducted by metro/core operators show about $73 \%$ of the traffic granularity will be 1 Gbps till 2020 [1]. Variety of implementations methods differentiate sub-wavelengths switching technologies; from node structure and sys-system component aspects relying on active or passive/reconfigurable components to the resource allocation methods and control plane protocols implementing routing and reservation based algorithms. Amongst the above-mentioned technologies Time-domain Wavelength Interleaved Networking (TWIN) [2], is an attractive solution as it offers optically passive sub-wavelength grooming and multiplexing capacity at intermediate nodes, as the intelligence and processing power remains at the edge nodes.

In this paper we present, for the first time to our knowledge, the implementation of the control plane and the TWIN edge node functionality within the recently proposed MEET network architecture for metro network [3].

\section{MULTI-HEAD SUB-WAVELENGTH SWITCHING (MEET) ARCHITECTURE FOR METROPOLITAN AREA NETWORKS (MAN)}

The metro level of a network operator connects the access networks and the operator customers (behind EN edge nodes) to the core networks (beyond $\mathrm{CN}$ concentration node) where connectivity to Internet, other service providers, content providers and service servers is usually provided. Fig. 1 (a) shows the three main interconnection points permitted through the $\mathrm{CN}$ : to the national/international core network Regional Nodes (RNs) (providing connectivity to other customers and to other local operators), to Internet Nodes (INs) (gateways of international network operators or large content providers) and to the operator services such as Video on Demand and VoIP hosted in Multiservice Nodes (MNs). The interconnection point between the metro network and the core network $(\mathrm{CN})$ is thus a node that must support large volumes of traffic and consequently large buffering and processing capacities, which may become problematic with the foreseen traffic volume growth.

In order to mitigate the problem at $\mathrm{CN}$ we proposed the Multi-hEad sub-wavElength swiTching, MEET architecture based on TWIN technology for metro networks [3]. Fig. 1 (b) presents a solution to replace the electronics buffers and concentration/distribution functions by one or several optical passive intermediate nodes that offer direct optical connections between ENs and RN/IN/MN in the core network.

We propose TWIN to enable the optical transparent concentration/distribution functions in MEET. According to TWIN architecture, each destination is assigned one (or several) wavelength(s). Correspondingly, to transmit data from several sources to one destination, one (or several) multiple-point to point trees are created where the destination is the root of the tree(s). The construction of the trees is out of the scope of this paper. We only assume that it is quasi-static however reconfigurable, the configuration of the optical nodes is based on 
multiplexers or wavelength selective switches. A source sends its traffic in the form of bursts of packets to the desired destination by tuning its transmitter to the wavelength corresponding to the destination. Deploying appropriate scheduling algorithm at the control layer ensures contention-free scheme. A schedule defines the time allocation pattern containing the succession of wavelengths (i.e. destinations) towards which each source must send the burst. This pattern can be changed or updated according to the traffic matrix at each Control Cycle(s) (CC).

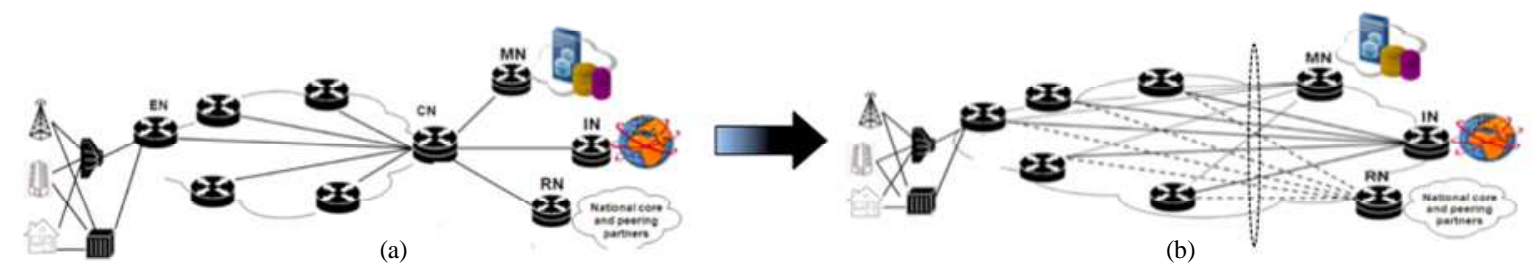

Figure 1. (a) Current metro logical topology, (b) Multi-hEad sub-wavElength swiTching Architecture.

\section{CONTROL PLANE ARCHITECTURE}

The notion of Real-Time control that is used here mostly emphasises on the compliance and functionality of the orchestration operations in a certain timing constraints. This characteristic is the key for critical systems that necessitate absolute consistency and reliability in time.

The scheduling algorithm is performed in the Central Control Entity (CCE), based on the traffic matrix and resource demands per source-destinations.

Therefore CCE needs to have the information concerning the traffic matrices, physical topology of the network with propagation delays between all links.

We assume that the operators estimate the traffic matrices and dimension the network according to its customers' demands (taking into account resiliency and quality of services constraints). In addition the propagation delays are also provided by the operators (for example measured during installation or during operation by specific measurement process). The Topology Discovery section (TD), keeps the propagation delays table updated in the CCE for scheduling purposes. The CCE and the nodes communicate via a control channel: the CCE sends allocation while the nodes send dynamic updates regarding changes in the link(s)/node(s) status and for example propagation delay updates.

At CCE all the above-mentioned information is combined and fed to the Compile and Schedule section (CaS). The CaS computes the scheduling file according to a given algorithm such as in [3]. Table 1, shows the grant files with one hundred $5 \mu \mathrm{s}$ time slots per cycle for source 1 and 2 that are implemented in this experiment.

\begin{tabular}{|c|c|}
\hline S1 & 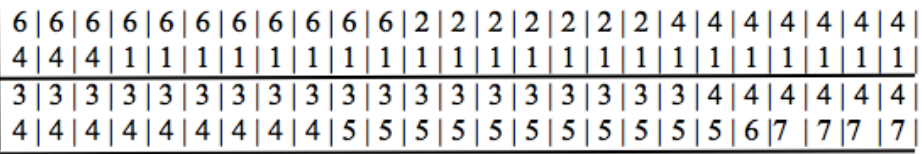 \\
\hline $\mathrm{S} 2$ & $\begin{array}{l}0|0| 0|0| 0|0| 0|0| 0|0| 0|6| 4|4| 4|4| 6|6| 2|2| 6|0| 5|5| 0 \mid \\
0|0| 0|0| 0|0| 0|7| 0|0| 3|3| 3|3| 3|0| 0|0| 0|0| 0|0| 0|0| 0 \mid \\
1|1| 0|0| 0|0| 0|0| 0|0| 0|0| 0|0| 0|0| 0|0| 0|0| 0|0| 0|0| 0 \mid \\
0|0| 0|0| 0|0| 0|0| 0|0| 0|0| 0|0| 0|0| 0|0| 0|0| 0|0| 0|0| 0 \mid\end{array}$ \\
\hline
\end{tabular}

Table 1. Grant file for one cycle (100 slots) for source 1 and source 2. The numbers indicate the wavelengths (i.e. destinations).

As a result the scheduling files are produced each corresponding to one source node within the network; these files are then sent via the control channel.

Each source is in charge of the burst assembly and burst emission (Real Time RT section) within the allocated time slots according to its Class of Service policies.

\section{EXPERIMENTAL SET-UP AND RESULTS}

In our previous studies we showed the interest of TWIN within MEET architecture for metro networks [3].

Fig. 2 (a) and (b) illustrate the schematic of the experimental setup. Fig 2 (a) presents the source and control parts and the passive core node is shown in Fig. 2 (b). We have considered 2 heads MEET architecture with the heads acting only as sources and $7 \mathrm{EN}$ acting only as destinations. 
Source-destination distances and traffic are set according to a French metro network.

In a real network a GPS master clock would be used to provide the required clock reference. In our set-up, a clock generator emulates this for all parts of the test-bed.

The test-bed contains:

The CCE: the CCE functions are realized thanks to a computer for CaS and the National Instrument (NI) RealTime engine on the PXI 1082 chassis and NI PXIe 7962R module featuring Xilinx Virtex-5 SXT FPGA for the RT functions.

The source: this part includes an Anritsu MT 1810A Pulse Pattern Generator (PPG) that operates in sequence mode and generates bursts of data with $4.7 \mu$ s duration. The guard-time between the bursts is set to $300 \mathrm{~ns}$ at emission. It takes care of possible clock inaccuracy and of tuning speed of the emitter. An additional $200 \mathrm{~ns}$ preamble is generated in front of the burst for burst reception clock recovery. Thus, each $5 \mu \mathrm{s}$, a burst can be sent according to scheduling file with grants. The PPG output electrical signal at $10 \mathrm{Gbps}$ is then printed on the optical signal generated by the Finisar S7500 Modulated Grating Y laser (MG-Y) tunable laser. Fast (less than $100 \mathrm{~ns}$ ) wavelength change on a burst per burst basis is assured by a fast driving board.

The $C N$ : it contains two fixed demultiplexers and two optical passive couplers.

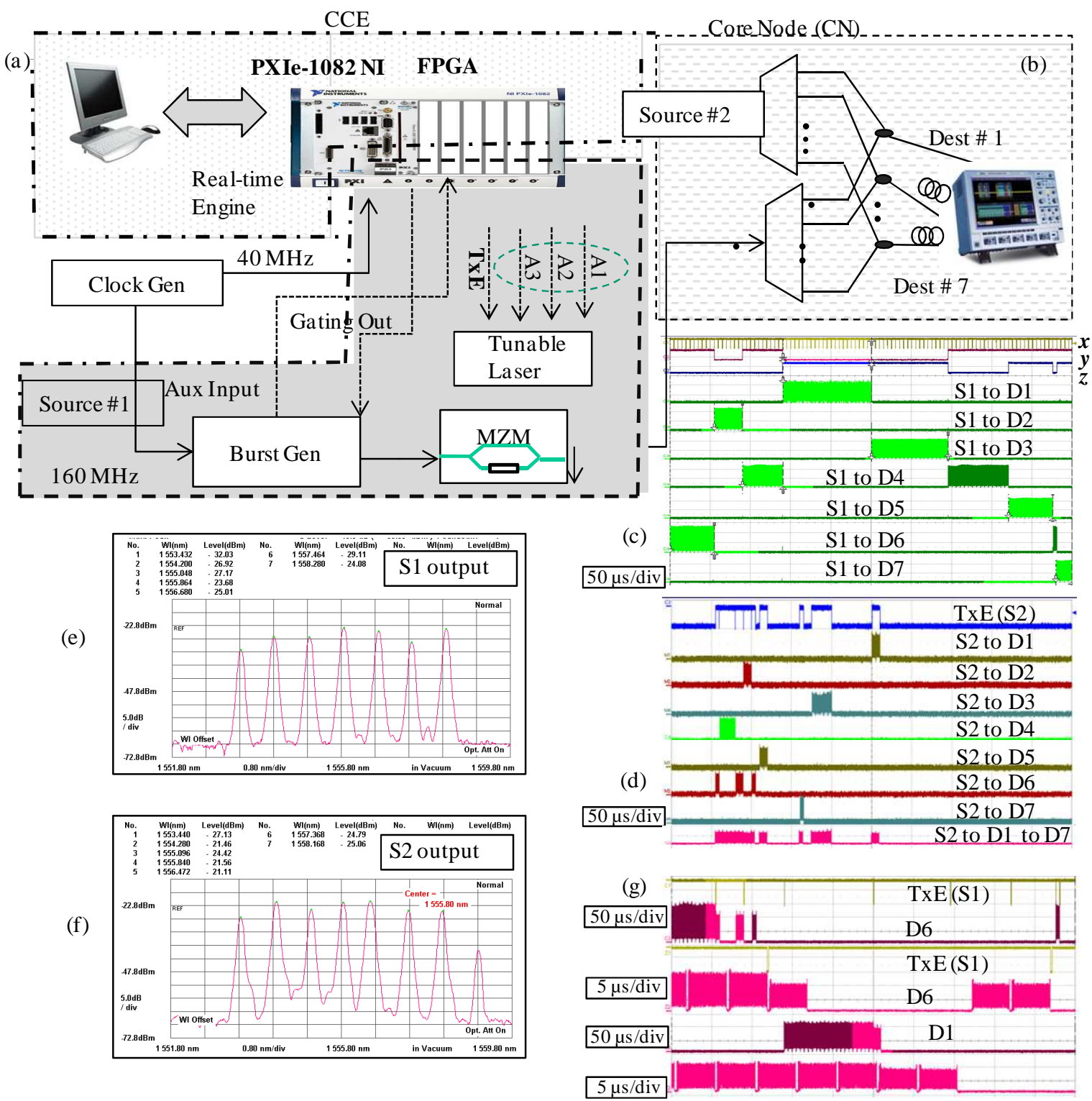

Figure 2. (a) Source and Central Control Entity setup section with central clock generator, (b) Core node with two demultiplexers and two couplers as the passive Core Node, $(C N),(c)$ Bursts transmitted by source 1 to seven destinations, (d) Bursts transmitted by source 2 to seven destinations, (e) Output spectrum of source 1, (f) Output spectrum of source 2, $(\mathrm{g})$ Traffic from two sources combined towards destination 6 and destination 1 after the demultiplexers and the two couplers. 
In detail, the external clock provides global time reference to the PPG but not the phase synchronization with the CCE. To obtain the phase synchronization the PPG sends gating out (GO) signals at the rhythm of burst emission (with $200 \mathrm{kHz}$ frequency) to FPGA part of the CCE. The CCE is ready to start sending its grant as soon as it detects the GO signal. The scheduling files are downloaded in the RT engine and transferred to the FPGA in the source segment. The FPGA at the source side receives the schedules and accordingly sends out the Aux Input signal to the PPG to generate the granted traffic per destination. In addition FPGA sends the enabling command "TxE i" and the address bits (A1, A2, A3) signals to the tunable laser which tunes its wavelength to lambda "i" (with "i" from 1 to 7 one per each destination according the address bits it receives).

In Fig. 2 (c) and (d), we can see the bursts that are emitted by source 1 and 2 towards destinations 1 to 7 according to the grant files in Table 1. Each trace shows the bursts filtered by the demultiplexers towards the corresponding destinations. At the top of Fig. 2 (c) there are 3 traces (x, y, z) that represent respectively, the bursts transmission rhythm, and 2 bits of address to tune the laser to the desired wavelength. Fig. 2 (e) shows 7 wavelengths spectrum at the output of source 1, while Fig. 2 (f) displays 8 wavelengths at the source 2 output, the extra wavelength being used as stuffing data when no grant is allocated. Since the enabling command TxE is off during grant 0 , (no allocation time) the output power of the stuffing data is also lower than the rest and this wavelength is chosen to be filtered after demultiplexers, therefore it is not propagated into the network.

Finally Fig. 2 (g) shows the bursts combined from the two sources towards destination 6, after the passive CN (2 upper traces). There are 11 bursts that are coming from source 1 according to Table 1 and immediately after 1 burst from source 2, that is perfectly aligned, then after 4 empty slots 2 more bursts are sent from source 2 to destination 6. The 2 lower traces of Fig. $2(\mathrm{~g})$ are the bursts combined from the two sources towards destination 1, after the passive CN. There are first 28 empty slots, followed by 22 bursts that are coming from source 1 according to Table 1 and immediately after 2 bursts from source 2, that are also entirely aligned with the bursts coming from source 2 .

As it is clearly shown there is no contention and the two flows from two separate sources are perfectly combined respecting the $300 \mathrm{~ns}$ guard time between the bursts after the $\mathrm{CN}$ and they all arrive as scheduled by the CCE.

With this set up we demonstrate the implementation of a control plane with collision free operation at the intermediate passive node. The control plane is in charge of synchronization and timing of the command signals and functionalities at every level of the network for a TWIN based two heads MEET architecture. Clearly the method is scalable to more number of source nodes (heads) in the network. In addition there are no active functionalities for the $\mathrm{CN}$, thus the need for buffering and processing power are completely eliminated from the core nodes and are limited to edge nodes and CCE.

\section{CONCLUSIONS}

In this work, we demonstrated for the first time, the essential bricks for the orchestration of data communication in sub-lambda switching networks: synchronization and scheduling. The demonstration purely focused on implementation and composition of the key control functions. The method is bit-rate independent and also applicable to higher or mixed data rates. Such orchestration is a vital enabler to provide high capacity, flexible and dynamically rearrangeable bandwidth with on demand provision capability for future metro networks.

\section{ACKNOWLEDGEMENTS}

This work was partly funded by the DGCIS in the frame of the CELTIC-Plus project SASER-SaveNet.

\section{REFERENCES}

[1] Strongest project deliverable, http://www.ict-strongest.eu/upload-files/deliverables-2/d2-1-updated-36 (2011).

[2] I. Widjaja et al., "Light core and intelligent edge for a flexible, thin-layered, and cost-effective optical transport network," Comm. Mag., vol. 41, no. 5, p. S30 (2003).

[3] A. Triki et al., "Is It Worth Adapting Sub-Wavelength Switching Control Plane to Traffic Variations?," Proc. ONDM, Stockholm (2014). 